\title{
Max Bömer
}

\section{Compeon GmbH - Digitaler Finanzmarktplatz am Rhein}

Die Digitalisierung in Wirtschaft und Gesellschaft ist in der vergangenen Dekade in den Fokus der gesellschaftlichen Diskussion gerückt. Die Digitalisierung kann das Wachstum und die Produktivität hierzulande steigern, gleichzeitig entstehen neue Arbeitsplätze in innovativen Unternehmen, welche die Wettbewerbsfähigkeit Deutschlands verbessern können.

Auch die Finanzindustrie ist von der Digitalisierung betroffen. Hier sind in den letzten Jahren junge Finanztechnologieunternehmen („Fintechs“) entstanden, die durch den Einsatz neuer Technologien innovative Finanzprodukte anbieten. Im Mittelpunkt dieser Fallstudie steht die Düsseldorfer Compeon GmbH, welche einen digitalen Marktplatz für gewerbliche Finanzierungen entwickelt hat. Auf der einen Seite haben Unternehmen die Möglichkeit, auf dieser Internetplattform Finanzierungswünsche einzustellen. Auf der anderen Seite können die kooperierenden Finanzdienstleister hierzu Angebote abgeben, aus denen die Unternehmen das passende auswählen. Darüber hinaus bietet die Plattform auch Finanzvertrieben und Unternehmensberatern Potenziale durch die Finanzierungsvermittlung.

Da die Gründer der Compeon GmbH einen finanzwirtschaftlichen Hintergrund und keine klassische Ausbildung als Software-Entwickler absolviert haben, wurde die technische Entwicklung der Internetplattform bei der Gründung der Compeon GmbH im Jahr 2012 einer Agentur als externem IT-Dienstleister überlassen. Im Zuge der Etablierung fassten die Gründer später den weitreichenden Entschluss, eine eigene IT-Abteilung aufzubauen, um die Weiterentwicklung der Plattform in die eigene Hand zu nehmen und weitere IT-Entwicklungen umzusetzen.

Vor diesem Hintergrund analysiert die vorliegende Fallstudie die Umsetzung des IT-Insourcings am Beispiel der Compeon $\mathrm{GmbH}$. Zuerst wird die Fintech-Branche in Deutschland näher beleuchtet und die Unternehmensgeschichte erzählt. Der Hauptteil konzentriert sich auf die Umsetzung des IT-Insourcings und zeigt auf, welche Gründe und Herausforderungen für die Compeon $\mathrm{GmbH}$ mit dieser Entscheidung verbunden waren. Abschließend erfolgt eine Zusammenfassung der Analyseergebnisse. 


\section{Finanzwelt im Umbruch}

Mit Verbreitung des Internets und der Digitalisierung sind neue Geschäftsmodelle und Produkte entstanden, die nicht selten ganze Branchen grundlegend verändert haben. In der vergangenen Dekade sind in Deutschland besonders viele Innovationen und Neugründungen im Bereich der Finanzdienstleistungen entstanden. Hinter den Innovationen stehen junge Unternehmen, die als „Fintechs“ bezeichnet werden. Eins ihrer gemeinsamen Merkmale ist, dass sie Finanzdienstleitungen durch internetbasierte Apps und den Einsatz moderner Technologien (künstliche Intelligenz, Big-Data-Analyse, Blockchain etc.) anbieten.

Der technische Fortschritt, die internationale Finanzkrise ab 2008 und das damit eingebüßte Vertrauen gegenüber Banken gelten als zentrale Faktoren für die Entstehung der Fintech-Branche in Europa und den USA. Vor allem junge Kunden/innen wandten sich von etablierten Banken ab, um die benutzerfreundlichen und transparenten Apps der Fintechs auszuprobieren. In diesem Sinne treten viele Fintechs mit ihren disruptiven Geschäftsmodellen in direkte Konkurrenz zu klassischen Finanzdienstleistern. Zudem gibt es eine weitere Gruppe von Fintechs, die das bestehende Bankenangebot um Mehrwert- oder Zusatzleistungen ergänzt. Eine dritte Gruppe von Fintechs bündelt die Dienstleistungen verschiedener Banken in Form von Vergleichs- und Vermittlungsportalen. Zu dieser letzten Gruppe zählt die Compeon GmbH. In der Vermittlung von gewerblichen Finanzierungen gab es im Jahr 2018 neben der Compeon GmbH als Marktführer nur einen aktiven Wettbewerber mit einem vergleichbaren Geschäftsmodell.

Die zahlreichen Kooperationen zwischen Banken und Fintechs verdeutlichen, dass sie mehr als nur konkurrierende Wettbewerber sind. Auf der einen Seite stehen Fintechs, die über innovative Lösungen und agile Teams verfügen, um kurzfristig auf Marktveränderungen reagieren zu können - häufig fehlen ihnen aber die notwendigen Ressourcen, um schnell und erfolgreich zu wachsen. Auf der anderen Seite stehen Banken, die aufgrund ihrer Organisationsstruktur eher träge agieren, wenn es um die Entwicklung und Implementierung neuer Lösungen geht. Allerdings besitzen sie umfangreiche finanzielle Ressourcen, einen großen Kundenstamm und die notwendigen regulatorischen Befugnisse, um beispielsweise Konten zu führen oder Gelder zu bewegen. Vor diesem Hintergrund haben Kooperationen zwischen alten und neuen Finanzdienstleistern ein großes Synergiepotenzial. Im Jahr 2016 waren knapp 90 Prozent aller deutschen Kredit- 
institute bereits eine Kooperation mit einem Fintech eingegangen oder planten zumindest eine solche Zusammenarbeit in der nahen Zukunft. ${ }^{1}$

Im europäischen Vergleich hat Deutschland mit über 700 jungen Unternehmen im Jahr 2017 die zweitgrößte Fintech-Branche nach Großbritannien. Seit 2008 ist sie jährlich um durchschnittlich 33 Prozent gewachsen. Damit steht die Fintech-Branche bei der Anzahl der Gründungen an vierter Stelle in Deutschland. Zudem registrierten deutsche Fintechs eine steigende Anzahl an Nutzern. 2017 hatte ein Drittel der deutschen Internetnutzer indirekten Kontakt zu dem Produkt von mindestens einem Fintech aus dem B2C-Bereich. ${ }^{2}$ Die meisten Fintechs sind in den Großstädten Berlin, München, Hamburg und den Metropolregionen RheinMain und Rhein-Ruhr beheimatet. Insbesondere in der Bundeshauptstadt hat sich durch das wachsende Start-up-Ökosystem und die hohen Venture-Capital-Investitionen in den vergangenen Jahren eine pulsierende Fintech-Szene entwickelt.

Als Nachteil für den Fintech-Standort Deutschland führen einige Experten die regulatorischen Hürden an. Für das Betreiben der meisten Finanzdienstleistungen benötigen Unternehmen in Deutschland nach dem Kreditwesengesetz oder anderen Normen eine kostspielige Lizenz. Im Unterschied zu Deutschland haben mehrere Länder wie Großbritannien, die Schweiz oder Singapur besondere regulatorische Rahmenbedingungen geschaffen, um Fintech-Gründungen zu fördern. Die sogenannten regulatorischen Sandkästen erlauben Fintechs, ihr Geschäftsmodell für einen bestimmten Zeitraum (zum Beispiel sechs Monate in Großbritannien) auszuführen, ohne die gewöhnlichen regulatorischen Vorgaben der Finanzaufsicht einhalten zu müssen.

Die Brexit-Entscheidung der Briten kann ein Wachstumstreiber für den deutschen Fintech-Markt sein, da Fintechs in Großbritannien Gefahr laufen, den Anschluss an den EU-Binnenmarkt zu verlieren. Bisher war es aufgrund der EU-Zugehörigkeit für britische Fintechs möglich, ihre Dienstleistungen ohne besondere Einschränkungen in Kontinentaleuropa zu vertreiben. Nach dem Wegfall der EUMitgliedschaft könnten einige britische Unternehmen ihren Hauptsitz von London nach Deutschland verlagern. In diesem Fall würden insbesondere deutsche Fintech-Hochburgen wie Berlin profitieren.

1 Vgl. Bundesfinanzministerium (2016), verfügbar unter https://www.bundesfinanzministerium. de/Content/DE/Standardartikel/Themen/Internationales_Finanzmarkt/2016-11-21-GutachtenLangfassung.pdf?_blob=publicationFile\&v=3.

2 Vgl. Digital Pioneers (2018), verfügbar unter https://t3n.de/news/fintech-Start-upsdeutschland-1097615/. 


\section{Gründer, die sich verstehen}

Die Compeon GmbH wurde 2012 von Dr. Frank Wüller, Kai Böringschulte und Dr. Nico Peters in Emsdetten (Nordrhein-Westfalen) gegründet. Neben der beruflichen Tätigkeit in ihrem Unternehmen verbindet die Gesellschafter eine jahrelange Freundschaft: Alle drei stammen aus dem Münsterland und kennen sich bereits seit dem Grundschulalter. Eine weitere Gemeinsamkeit der Gründer besteht darin, dass jeder von ihnen eine klassische Ausbildung bei einer Bank bzw. Sparkasse absolviert hat. Mit Beginn des Studiums trennten sich ihre Wege dann vorläufig.

Wüller studierte Betriebswirtschaft und internationale Finanzwirtschaft an der Fachhochschule Münster und der Graduate School Pforzheim, bevor er später an der Bergischen Universität Wuppertal promovierte. Böringschulte erlangte wissenschaftliche Abschlüsse in Volkswirtschaftslehre und Betriebswirtschaftslehre an der Fernuniversität Hagen. Peters schloss an der Universität Münster den Studiengang Betriebswirtschaftslehre mit den Schwerpunkten Finanzwirtschaft und Supply Chain Management ab und promovierte im Anschluss an der Bergischen Universität Wuppertal.

Nach ihren Abschlüssen arbeiteten die drei zunächst wieder in ihren Ausbildungsunternehmen, bevor sie in den Jahren 2006 und $2007 \mathrm{zu}$ einer bankorientierten Unternehmensberatung nach Münster wechselten, um dort im Firmenkundenbereich tätig zu werden. Zentrale Schwerpunkte waren prozessuale, vertriebliche und strategische Fragestellungen der gewerblichen Finanzierung. Darüber hinaus haben sie Banken und Sparkassen deutschlandweit beraten, mitunter über deutsche Grenzen hinaus.

Trotz der ähnlichen Lebensläufe macht Wüller deutliche Unterschiede hinsichtlich der Kompetenzen der Gründer aus: „Nico Peters ist bei uns der strategische Kopf, weil er sehr analytisch denkt. Kai Böringschulte ist sicherlich sehr stark im Vertrieb, und ich bin eher Generalist und kümmere mich außerdem um die Themen Finance und Operations. Das waren auch damals schon unsere Rollen bei der ZEB und die haben wir auch bei Compeon fortgeführt.“

\section{Am Anfang war die Idee}

Auf die Geschäftsidee, die Compeon GmbH zu gründen, ist das Trio im Jahr 2010 aufgrund seiner Beratertätigkeit für Banken gekommen. Im Rahmen von Projekten bearbeiteten die drei viele Fragestellungen zum Firmengründergeschäft. Dabei hatten sie auch Einblicke in den Privatkundenmarkt, der sich zu dieser Zeit durch die Digitalisierung rasant veränderte. Wüller erinnert sich: „Für uns lag 
der Schluss nahe, dass die Digitalisierung beim Firmenkundengeschäft nicht Halt machen würde. Die Ideen spielten sich in dem Umfeld Digitalisierung, Firmenkundengeschäft und Kundengewinnung bzw. Kundenbindung ab. Daher sind die Gedanken aufgekommen, dass wir Spaß daran haben würden, uns selbstständig zu machen. Es stand natürlich die Frage im Raum, wie wir dies konkret machen. Unterschiedliche Recherchen, Projekte, Interviews und Ähnliches haben uns dann letztlich dazu veranlasst, ein Marktplatzmodell für gewerbliche Finanzierungen ins Leben zu rufen, und das haben wir dann Ende 2012 mit der Gründung umgesetzt.“

Als Vorbild dienten diverse digitale Marktplätze aus verschiedenen Branchen und Industrien. Ein spezielles Marktplatzmodell aus dem Bereich Finanzdienstleistungen, welches sich die Gründer sehr genau angeschaut hatten, war interhyp. Darüber hinaus beschäftigten sich die drei Gründer auch grundsätzlich mit dem Thema Marktplatzökonomie und gingen der Frage nach, wie Marktplätze eine höhere Effizienz erzielen können. Dafür untersuchten sie die großen strukturierteren und standardisierten Marktplätze wie Check24, Amazon oder Airbnb.

\section{Die ersten fünf Jahre}

Nach dem Markteintritt im Jahr 2013 hat die Compeon GmbH es geschafft, das Anfragevolumen und den Umsatz jedes Jahr zu verdoppeln. Bislang wurden bereits mehr als 500 Millionen Euro an Finanzierungsvolumen erfolgreich bei Banken platziert. Für das Jahr 2019 strebt die Compeon GmbH die Überschreitung der 1-Milliarden-Grenze an. Wüller erklärt das rasante Wachstum mit zwei Effekten: „Zum einen gibt es den Effekt, dass wir durch die steigende Größe mehr Marketing machen und dadurch präsenter sind. Der zweite Effekt ist, dass der Markt für online vermittelte Finanzierung ein sehr stark wachsender Markt ist. Der Marktwachstumseffekt erklärt sich durch einen Wandel von der stationären 1:1-Hausbankbeziehung hin zu unabhängigen Ansätzen. Unsere Wachstumskurve flacht dadurch nicht ab, sondern gewinnt an Dynamik.“

Der Erfolg der Compeon $\mathrm{GmbH}$ lässt sich unter anderem an der Mitarbeiteranzahl ablesen. Nach dem Start mit den drei Gründern stieg in den folgenden zwei Jahren die Anzahl der Mitarbeiter/innen langsam auf zehn. Im Sommer 2018 umfasste das Team bereits etwa 80 Mitarbeiter/innen in den Bereichen IT, Marketing, Firmenkundenbetreuer und Administration. Anfang 2019 ist die Zahl der Mitarbeiter auf mehr als 100 gestiegen. 


\section{Was Insourcing mit dem Standort zu tun hat}

Die wichtigste Entscheidung der Gründer für ihr Unternehmen war der Aufbau einer eigenen IT-Abteilung, um die Compeon-Plattform vollständig unabhängig von externen Dienstleistern betreuen zu können, nachdem mit der Entwicklung der Plattform zunächst eine Agentur beauftragt worden war.

Im betriebswirtschaftlichen Kontext spricht man an dieser Stelle von „Insourcing “, das heißt, zuerst wird eine unternehmerische Aktivität an ein anderes Unternehmen ausgelagert (Outsourcing), welche dann in einer späteren Unternehmensphase internalisiert wird. Es gibt verschiedene Gründe, warum Unternehmen zunächst die IT auslagern. Meistens möchten sie sich zuerst auf andere Hauptanliegen konzentrieren. Im Fall der Compeon $\mathrm{GmbH}$ stand zunächst die Gewinnung von Banken im Vordergrund. Wüller dazu: „Der Kern unserer Tätigkeit war zu Beginn von Compeon die Bankengewinnung. Diese lag sehr stark bei uns Gründern, da wir viele Banken aus unserer alten Tätigkeit kannten. Schätzungsweise 80 Prozent der Zeit, die wir täglich allokiert haben, wurde für die Partnergewinnung investiert. Wir haben uns damit auf die Angebotsseite des Marktplatzes konzentriert. Das Kundenmarketing und die IT waren damals noch ausgelagert.“

Ein weiterer Grund für die initiale Auslagerung der IT war, dass die Gründer eher Kompetenzen auf den Gebieten Betriebs- und Finanzwirtschaft besitzen und keine Programmierer sind.

Im Laufe einer Unternehmensentwicklung beginnen viele Gründer, die Auslagerungssituation kritisch zu hinterfragen. Grundsätzlich bestehen verschiedene Ursachen, warum Insourcing in einer späteren Unternehmensphase attraktiv wird:

1. Geschwindigkeit und Flexibilität:

Vor allem im IT-Bereich sind schnelle Reaktionszeiten und eine hohe Flexibilität von hoher Bedeutung. Eine interne Entwicklung ermöglicht eine schnellere und effizientere Kommunikation als zu einem externen Entwickler. Hier kommt es häufig zu Rückfragen und langen Umsetzungs- bzw. Entwicklungszeiten.

2. Wiedererlangen von Know-how:

Mit der Outsourcing-Entscheidung ist gleichzeitig ein Verlust von firmeninternem Know-how verbunden. Dies ist vor allem dann kritisch, wenn es um das sensible Kerngeschäft von Unternehmen geht.

3. Reduzierung der Abhängigkeit:

Durch die Auslagerung von Prozessen begibt sich das Unternehmen in eine Abhängigkeit von externen Anbietern. 
4. Arbeitsmoral und -loyalität:

Mitarbeiter/innen, die sich stark mit einem Unternehmen und dessen Produkten identifizieren, liefern oft bessere Ergebnisse als solche, die keinen unmittelbaren Bezug zu dem Unternehmen haben.

5. Kontrolle und Sicherheit:

Erfolgt die Produktion intern, hat die Geschäftsführung eine bessere Übersicht und Kontrolle der Projekte. Sie wird jederzeit über Fortschritte informiert und kann ggf. Maßnahmen ergreifen, falls Probleme auftreten. Die direkte Kontrolle einer internen Produktion schützt das Unternehmen besser gegen Verbrechen, Diebstahl, Angriff, Sabotage und Spionage.

6. Höhere Servicequalität und Kundenzufriedenheit:

Die interne Produktion kann zudem die IT-Servicequalität und Kundenzufriedenheit steigern, da die tatsächliche Qualität der ausgelagerten Produktion mitunter durch standardisierte Lösungen aufseiten des Anbieters nicht den Kundenerwartungen entspricht.

7. Kostenreduktion:

Outsourcing dient dazu, Kosten zu senken. Falls jedoch standardisierte Lösungen zu einer unerwartet schlechten Servicequalität führen, kommt es wiederum verstärkt zu Änderungswünschen, welche mit einem deutlichen Kostenanstieg verbunden sind.

Die entscheidenden Motive für das Insourcing der IT waren im Fall von Compeon eine Steigerung von Geschwindigkeit und Flexibilität, um die Entwicklungszyklen zu verkürzen und die Umsetzungszeiten zu verringern.

Insourcing hat für Unternehmen in bestimmten Situationen viele Vorteile. Allerdings sind verschiedene Herausforderungen und Risiken mit dieser Entscheidung verbunden, welche unbedingt berücksichtigt werden sollten:

1. Preis:

Unternehmen neigen bei der Planung des Insourcings dazu, den Business Case „schön zu rechnen“. Dabei passen sie häufig die bevorstehenden Kosten für die interne Umsetzung so an, dass sie unterhalb der Kosten des externen Servicepartners liegen. Dies birgt allerdings die Gefahr, dass Abstriche hinsichtlich Umsetzungszeit und Qualität notwendig sind.

2. Übergabe:

Auch bei und nach der Übergabe des Projekts kann es zu unangenehmen Überraschungen kommen, wenn Rechte und Pflichten im Zusammenhang mit der extern hergestellten Leistung in der Exit-Vereinbarung nicht eindeutig oder zum Nachteil des Unternehmens geregelt sind. Es kann zum Beispiel sein, dass bestimmte Lizenz- oder Verfügungsrechte an dem Projekt bei dem 
externen Dienstleister verbleiben und somit eine Abhängigkeit bestehen bleibt.

3. Betriebsblindheit:

Nachdem das Projekt internalisiert wurde, besteht die Gefahr, dass keine oder eine nur unzureichende objektive Einschätzung im Hinblick auf die eigenen Leistungen stattfindet. So werden einfache Fehler übersehen und alternative Lösungsansätze außer Acht gelassen. Beides kann in letzter Konsequenz zu hohen Kosten und einer gehemmten Innovationsfähigkeit des Unternehmens führen.

4. Personal:

Der zentrale Punkt beim Insourcing ist der Aufbau eines Teams, welches die Aufgaben des zuvor ausgelagerten Projekts übernimmt. Hierfür muss genügend Personal mit zeitgemäßen IT-Fachkenntnissen zur Verfügung stehen. Nur dann ist eine erfolgreiche Übernahme des Projekts gewährleistet. Steht nicht ausreichend qualifiziertes Personal zur Verfügung oder sind weitere benötigte Ressourcen (Hardware-Ausstattung, Server-Kapazität, SoftwareLizenzen etc.) nicht korrekt geplant, kann es zu kostspieligen Engpässen kommen. Teilweise versuchen Unternehmen dann, bestimmte Aktivitäten anderen Abteilungen zu übergeben, um den eigenen Aufwand zu senken, was allerdings $\mathrm{zu}$ unübersichtlichen und schwer handhabbaren Prozessen führt. Darüber hinaus müssen Unternehmen gewährleisten, dass Mitarbeiter/ innen mit den schnelllebigen Entwicklungen in der IT Schritt halten. Dafür müssen sie laufend weitergebildet und geschult werden, wodurch zusätzliche Kosten entstehen.

\section{Standortwechsel - gut für das Personal}

Für die Compeon $\mathrm{GmbH}$ bestand die größte Herausforderung beim Insourcing der IT darin, ein eigenes IT-Team aufzubauen, welches die Arbeitsfelder der Agentur übernahm und fortführte. Dabei zog die Entscheidung des IT-Insourcings eine weitere Schlüsselfrage nach sich.

Die Compeon GmbH wurde in Emsdetten, einer Kleinstadt mit etwa 36.000 Einwohnern, gegründet. Bei dem Versuch, dort ein eigenes IT-Team aufzubauen, stellten die Gründer fest, dass sie im Münsterland zwar Bankkaufleute gut rekrutieren konnten, allerdings keine qualifizierten IT-Fachkräfte. Wüller erinnert sich: „Als wir begonnen haben ITler zu suchen, die Know-how im Online-Marketing haben und sich in der Nische Internet und Finance auskennen, mussten wir schnell feststellen, dass nur sehr wenige Bewerbungen eingingen. Hinzu kam, dass die 
Bewerber an Homeoffice-Ansätzen interessiert waren, weil sie sich nicht vorstellen konnten, nach Emsdetten zu ziehen.“

So wurde den Gründern klar, dass ein neuer Standort für die Compeon GmbH benötigt wird, um eine schlagkräftige eigene IT-Abteilung aufbauen. Es kamen verschiedene Standorte infrage. Um herauszufinden, welche Städte am besten für den Aufbau des IT-Teams geeignet sind, veröffentlichten die Gründer Stellenanzeigen für verschiedene Standorte und warteten das Feedback ab. Wüller dazu: „Wir stellten fest, dass Düsseldorf bei den Bewerbern gut ankam. Wir haben uns dann dazu entschieden, den kleinen Standort, der bereits in Düsseldorf bestand, stetig zu erweitern. Also korrelierte der Teamaufbau der IT stark mit dem Umstand, dass wir nach Düsseldorf gegangen sind.“

Die drei Gründer fanden in Alex Bierhaus einen IT-Experten als Leiter des IT-Teams, der zuvor schon im Raum Düsseldorf verortet war und als neuer vierter geschäftsführender Gesellschafter den Aufbau der internen IT-Abteilung maßgeblich vorantrieb. Wüller erinnert sich: „Alex Bierhaus ist bei unterschiedlichen Events aufgetreten und hat Compeon bekannter gemacht. Dadurch haben wir es geschafft, sehr gute Entwickler, die am Markt rar gesät sind, für Compeon zu gewinnen.“

Die Landeshauptstadt unterscheidet sich von anderen Start-up-Metropolen wie Berlin laut Wüller in der geringeren Arbeitnehmerfluktuation, insbesondere bei IT-Fachkräften. Außerdem ist eine hohe Anzahl an IT-Fachkräften durch die breit gefächerte Unternehmenslandschaft und die verschiedenen Hochschulen in Düsseldorf unmittelbar beheimatet. Zudem hat die Stadt eine gewisse Strahlkraft und Fachkräfte sind gewillt, für einen neuen Job nach Düsseldorf umzuziehen. Wüller dazu: „Wir haben einige Kollegen gewinnen können, die aus der Region Münster kommen und es als spannendes Momentum gesehen haben, jetzt mit einer Jobveränderung nach Düsseldorf zu ziehen.“

\section{Standortwechsel - gut für Kunden}

Die Entscheidung des IT-Insourcings ging mit einem Wechsel des Standorts von Emsdetten nach Düsseldorf einher, da dort eine bessere Personalverfügbarkeit von IT-Fachkräften gegeben ist. Darüber hinaus brachte der neue Standort weitere wichtige Vorteile mit sich, die sich positiv auf das Wachstum der Compeon GmbH auswirkte.

Die Compeon GmbH spricht mit ihrem Marktplatz für die Vermittlung von gewerblichen Finanzierungen mehrere unterschiedliche Zielgruppen an. Zum einen handelt es sich um Unternehmen, die über die Plattform wegen einer Finanzierung anfragen. Zum anderen sind die angeschlossenen Finanzdienstleister eben- 
falls als Kunden anzusehen, da die Compeon $\mathrm{GmbH}$ durch das Marktplatzmodell von ihnen nach erfolgreicher Vermittlung eine Vergütung erhält und mit der Anzahl an Finanzdienstleistern das Angebot für die Unternehmen wächst und die Plattform attraktiver wird. Des Weiteren bietet die Plattform auch Potenzial für Finanzbetriebe und Berater, die durch die Plattform Finanzierungsvorhaben ihrer gewerblichen Kunden ausschreiben. Auch Unternehmen nutzen Compeon für den Absatz ihrer Produkte, um ihren Kunden so direkt die passende Finanzierung zum Produkt liefern zu können.

Im Hinblick auf die Firmenkunden ist Düsseldorf als neuer Standort für die Compeon GmbH interessant, da die Stadt geostrategisch im Rheinland und in der Nähe des Ruhrgebiets liegt. Aufgrund dieser Lage haben sich dort viele Firmen aus unterschiedlichen Industrien angesiedelt, was ebenfalls bei der Standortentscheidung berücksichtigt wurde. Wüller erklärt: „In NRW findet man im europäischen Vergleich die größte Akkumulation von mittelständischen Unternehmen vor. Mit unserem Business-to-Business-Konzept war das ein sehr wichtiger Faktor in unserer Standortentscheidung."

Über die digitale Plattform der Compeon GmbH schließen Kunden in ganz Deutschland online Finanzierungen ab. Daher nehmen die Kundenbetreuer des Unternehmens selten Termine außer Haus wahr. Allerdings stellt Wüller fest, dass eine gewisse räumliche Nähe einen positiven Effekt auf Wahrnehmung der Firmenkundschaft ausübt.

Darüber hinaus wurde die Dichte an Finanzinstituten bei der Standortwahl berücksichtigt. In Deutschland stellt Frankfurt am Main den Hauptfinanzmarktplatz dar. Im Fall der Compeon $\mathrm{GmbH}$ war dies allerdings nicht ausschlaggebend. Wüller dazu: „Frankfurt ist zwar der größte Finanzplatz in Deutschland. Aber von unseren zentralen Partnern sitzen gar nicht mehr so viele in Frankfurt. Wir setzen sehr bewusst darauf, mit den Primärinstituten wie Volksbanken, Raiffeisenbanken und Sparkassen zusammenzuarbeiten. Darüber hinaus sitzen aber auch die großen Privatbanken oder auch kleinere Privatbanken in anderen Städten als Frankfurt. Düsseldorf bietet daher mit der zentralen Lage für uns weitere Vorteile.“

\section{Kurz und bündig}

Die Digitalisierung betrifft jeden, da sie mit einem tiefgreifenden Wandel in nahe$\mathrm{zu}$ jedem Lebensbereich verbunden ist. Die digitale Transformation in Deutschland birgt große Chancen für mehr Lebensqualität, neue Geschäftsmodelle und effizienteres Wirtschaften. 
Inzwischen hat die Digitalisierung in Deutschland auch die Finanzindustrie eingeholt. Junge Fintechs überraschen mit innovativen Ideen und sorgen für Aufbruchsstimmung. Einige von ihnen werden zu einer Gefahr für etablierte Banken und Versicherungen, da ihre Dienstleistungen mögliche Alternativen für Kunden darstellen. Andere Fintechs arbeiten bewusst mit Banken zusammen und nutzen Synergiepotenziale.

Eins der erfolgreichsten Fintechs in Deutschland ist die Compeon $\mathrm{GmbH}$. Über ihre Internetplattform für gewerbliche Finanzierungen bringt sie Firmenkunden, die Finanzierungen nachfragen, und Finanzpartner, die Finanzierungslösungen anbieten, zusammen. Auf diese Art und Weise eröffnet das Unternehmen den Banken einen neuen Vertriebsweg und bietet den Firmen eine transparente Auswahl an Finanzierungsangeboten.

Wie bei den meisten Fintech-Gründungen kam auch die Idee für die Compeon $\mathrm{GmbH}$ von erfolgreichen Branchenkennern, hier Beratern, die auf eine jahrelange Berufserfahrung im Firmenkundengeschäft zurückblickten. Nach einer intensiven Auseinandersetzung mit gewerblichen Finanzierungen erkannten die drei Gründer, dass digitale Marktplätze auch im Bereich der Mittelstandsfinanzierung zu einem erheblichen Effizienzgewinn führen können. Die technische Umsetzung des digitalen Geschäftsmodells erfolgte zunächst wie bei den vielen Fintechs durch eine beauftragte Agentur.

Die externe Entwicklung einer digitalen Plattform ist häufig die zunächst beste Lösung für Fintech-Gründer, da diese - wie im Fall der Compeon GmbH - aus der Finanzindustrie stammen und keine Software-Entwickler im klassischen Sinne sind. Die externe Betreuung der Plattform bringt allerdings auch diverse Nachteile mit sich. Da digitale Geschäftsmodelle permanent auf dem Prüfstand stehen und laufend weiterentwickelt werden müssen, entschieden sich die Gründer dafür, eine eigene IT-Abteilung aufzubauen, um die Plattform schnell und flexibel pflegen zu können. Darüber hinaus entwickeln die IT-Mitarbeiter neben der zentralen Plattform auch weitere Service-Angebote für Kunden wie einen Chatbot, Voice Assist oder die Integration in Partnersysteme über tiefe Schnittstellen.

Im Zuge dieser Umstrukturierung bemerkten die Gründer bald, dass an ihrem ursprünglichen Gründungsort Emsdetten nicht ausreichend Programmierer vorhanden waren und Programmierer außerhalb der Stadt nicht überzeugt werden konnten, in die Kleinstadt zu wechseln. Daher zog die Entscheidung des ITInsourcings eine Verlegung des Standorts nach Düsseldorf nach sich, da die Verfügbarkeit von gut ausgebildeten IT-Spezialisten in der Rheinmetropole deutlich besser aussah. Zudem befindet sich die Compeon $\mathrm{GmbH}$ nun näher an vielen Firmenkunden und Banken, welche zu einem großen Teil im Ballungsgebiet RheinRuhr verortet sind. 
Infolge der IT-Eingliederung haben sich die Entwicklungszyklen für die Plattform deutlich verkürzt. Die Compeon $\mathrm{GmbH}$ ist inzwischen deutlich schneller und flexibler bei der Entwicklung und Implementierung neuer Features. Die höhere Agilität in der Plattformentwicklung übt zudem einen unmittelbaren Einfluss auf die Anzahl der Anfragen aus. Damit ist das Unternehmen nun bestens aufgestellt, um weiterhin zu wachsen und die Rolle des Marktführers in Deutschland in der digitalen Vermittlung von gewerblichen Finanzierungen zu behaupten. 


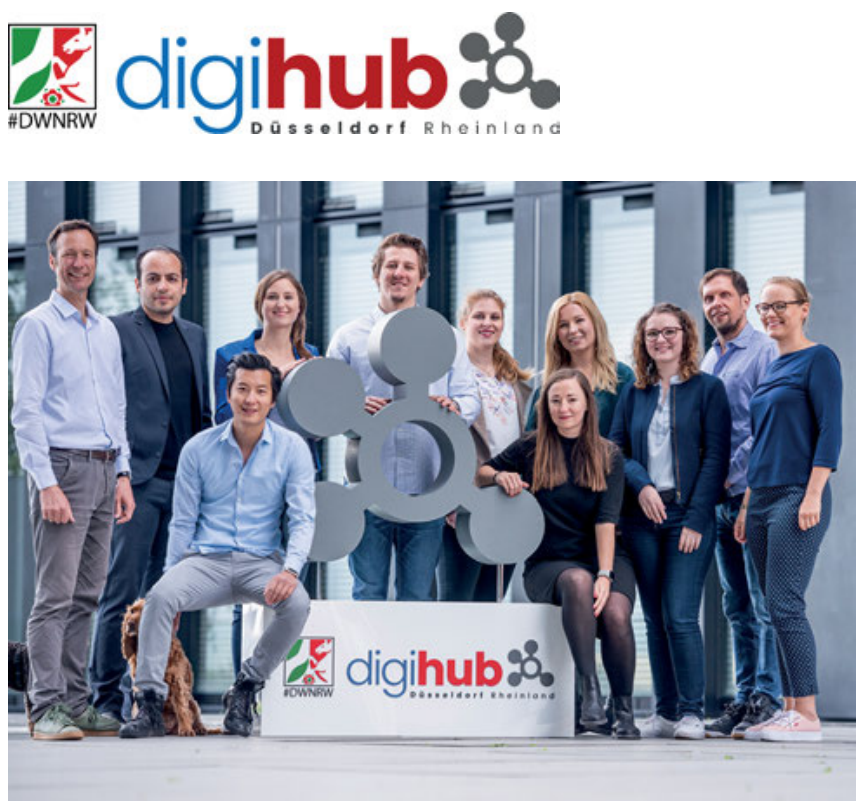

Das Team des digihub Düsseldorf/Rheinland

(Bildnachweis: digihub Düsseldorf/Rheinland) 